\title{
Phase Pure Synthesis and Morphology Dependent Magnetization in Mn Doped ZnO Nanostructures
}

\author{
Murtaza Saleem, ${ }^{1}$ Shahid Atiq, ${ }^{2}$ Shahid M. Ramay, ${ }^{3}$ Asif Mahmood, \\ Saadat A. Siddiqi, ${ }^{5}$ and Muhammad Ali Shar ${ }^{6}$ \\ ${ }^{1}$ School of Science and Engineering (SSE), Lahore University of Management Sciences (LUMS), Opposite Sector U, D.H.A., \\ Lahore Cantt 54792, Pakistan \\ ${ }^{2}$ Centre of Excellence in Solid State Physics, University of the Punjab, Quaid-e-Azam Campus, Lahore 54590, Pakistan \\ ${ }^{3}$ Physics and Astronomy Department, College of Science, King Saud University, P.O. Box 800, Riyadh 11421, Saudi Arabia \\ ${ }^{4}$ Chemical Engineering Department, College of Engineering, King Saud University, P.O. Box 800, Riyadh 11421, Saudi Arabia \\ ${ }^{5}$ Interdisciplinary Research Centre in Biomedical Materials, COMSATS Institute of Information Technology, Defence Road, \\ Off Raiwind Road, Lahore 54600, Pakistan \\ ${ }^{6}$ Mechanical Engineering Department, Center of Excellence for Research in Engineering Materials, King Saud University, \\ P.O. Box 800, Riyadh 11421, Saudi Arabia
}

Correspondence should be addressed to Murtaza Saleem; murtaza.saleem@lums.edu.pk

Received 30 January 2014; Accepted 25 April 2014; Published 19 May 2014

Academic Editor: Weihua Tang

Copyright (C) 2014 Murtaza Saleem et al. This is an open access article distributed under the Creative Commons Attribution License, which permits unrestricted use, distribution, and reproduction in any medium, provided the original work is properly cited.

\begin{abstract}
$\mathrm{Zn}_{0.95} \mathrm{Mn}_{0.05} \mathrm{O}$ nanostructures were synthesized using sol gel derived autocombustion technique. As-burnt samples were thermally annealed at different temperatures $\left(400,600\right.$, and $\left.800^{\circ} \mathrm{C}\right)$ for 8 hours to investigate their effect on structural morphology and magnetic behavior. X-ray diffraction and scanning electron microscopic studies demonstrated the improvement in crystallinity of phase pure wurtzite structure of $\mathrm{Mn}$ doped $\mathrm{ZnO}$ with variation of annealing temperature. Energy dispersive X-ray elemental compositional analysis confirmed the exact nominal compositions of the reactants. Electrical resistivity measurements were performed with variation in temperature, which depicted the semiconducting nature similar to parent $\mathrm{ZnO}$ after 5 at $\% \mathrm{Mn}$ doping. Magnetic measurements by superconducting quantum interference device detected an enhanced trend of ferromagnetic interactions in thermally annealed compositions attributed to the improved structural morphology and crystalline refinement process.
\end{abstract}

\section{Introduction}

Diluted magnetic semiconductors (DMSs) have attracted a great deal of interest in recent years due to their applications in modern spintronic devices $[1,2]$. One of the major thrusts in ongoing research is the formation of phase pure DMS materials and achievement of ferromagnetism with reasonable magnetization [3, 4]. Theoretical investigations suggest that semiconductors with wide band-gaps are the most potential candidates for this purpose $[5,6]$. $\mathrm{ZnO}$ has a direct band-gap $\left(E_{g}=3.37 \mathrm{eV}\right)$ among semiconductors with hexagonal (wurtzite) crystal structure. Several investigations have been reported by various research groups to synthesize phase pure transition metals (TMs) doped $\mathrm{ZnO}$ DMSs [7-9]. In particular, $\mathrm{Mn}$ doped $\mathrm{ZnO}$ have been prepared to explore their potential applications in spintronic devices and biomedicine by exploiting their unique magnetic and antibacterial properties [10]. Theoretical prediction for ferromagnetism in $\mathrm{Mn}$ doped $\mathrm{ZnO}$ has attracted much experimental research interest among these $\mathrm{ZnO}$ based DMSs [11]. Many reports exist for achievement of ferromagnetism in these materials $[12,13]$. Some exciting results for $\mathrm{Mn}$ doped $\mathrm{ZnO}$ bulk compositions have been reported. Ferrimagnetic phase transition in $\mathrm{Zn}_{1-x} \mathrm{Mn}_{x} \mathrm{O}(x=0.05)$ sample was observed, which was reported due to the existence of spinel impurity phase like $\mathrm{Mn}_{2} \mathrm{O}_{4}$ in this system [14]. Li 
and coworkers [15] obtained ferromagnetism in $\mathrm{Zn}_{1-x} \mathrm{Mn}_{x} \mathrm{O}$ samples prepared and sintered in nitrogen atmosphere. Room-temperature ferromagnetism was achieved by Sharma et al. [16] in low temperature synthesized thin films and bulk samples of $\mathrm{ZnMnO}$ systems. Recently, sintering temperature effect on structural and magnetic properties of Mn doped $\mathrm{ZnO}$ bulk system has been studied $[17,18]$ and ferromagnetic interactions have been detected in a composition sintered up to $700^{\circ} \mathrm{C}$. Further, it is also observed that TMs doped $\mathrm{ZnO}$ exhibit morphology dependent magnetic behavior, wherein it is recommended that the improved morphology is responsible for enhanced magnetic ordering [19-21]. In addition, emission properties of $\mathrm{Mn}$ doped $\mathrm{ZnO}$ nanomaterials have also got the attention of scientific community [22]. These studies revealed that preparation technique, thermal annealing, and structural morphology directly influenced the various properties. In addition, the phase pure synthesis of DMS materials with achievement of enhanced magnetization is an essential objective for practical applications in emerging spintronic devices. Hence, this research work is a unique attempt to enhance the magnetic behavior in $\mathrm{Mn}$ doped $\mathrm{ZnO}$ DMS through improved structural morphology.

The sol gel autocombustion technique offers distinctive synthesis advantages. This ensures the oxide formation and crystallization to create the required and necessary phases of nanostructure materials in a short time [7]. In this work, nanostructures of $\mathrm{Zn}_{0.95} \mathrm{Mn}_{0.05} \mathrm{O}$ have been prepared using this technique and afterwards thermally annealed at various temperatures in order to observe their effect on different properties of prepared samples. Thermal annealing significantly affects the structural morphology of material which directly influences the electrical and magnetic properties. Moreover, extensive profound role of impurity phases formed at high-temperature thermal annealing treatment was also observed.

\section{Experimental Methods}

In order to prepare $\mathrm{Mn}$ doped $\mathrm{ZnO}$ nanostructures, molar ratios of $\mathrm{Zn}$ nitrate $\left[\left(\mathrm{Zn}\left(\mathrm{NO}_{3}\right)_{2} \cdot 6 \mathrm{H}_{2} \mathrm{O}\right], \mathrm{Mn}\right.$ nitrate $[(\mathrm{Mn}$ $\left.\left(\mathrm{NO}_{3}\right)_{2} \cdot 6 \mathrm{H}_{2} \mathrm{O}\right]$, and citric acid $\left(\mathrm{C}_{6} \mathrm{H}_{8} \mathrm{O}_{7}\right)$ with $1: 1$ metal nitrates to citric acid ratio were dissolved in distilled water. All reagents used were of analytical quality. The $\mathrm{pH}$ of the solution was maintained at 7 by adding the proper amount of liquid ammonia $\left[\mathrm{NH}_{3}\right]$. The solution was then constantly stirred and dried at $150^{\circ} \mathrm{C}$ to obtain xerogel. After attaining the xerogel, the temperature was increased to $250^{\circ} \mathrm{C}$ and the gel was converted into powder by self-combustion. The resulting as-burnt powder was annealed at 400,600, and $800^{\circ} \mathrm{C}$ for 8 hours in ordinary environment in a muffle furnace (Ogawa Seiki). As-burnt and thermally annealed samples were characterized by X-ray diffraction (XRD) using a Panalytical X'Pert Pro multipurpose diffractometer (MPD). The X-ray diffractometer was operated at $40 \mathrm{kV}$ and $40 \mathrm{~mA}$ with $\mathrm{Cu} K_{\alpha 1}$ radiation $(\lambda=1.540598 \AA)$ with step scan size of 0.02 . Field emission scanning electron microscopic studies were carried out using S-3700N (Hitachi Japan) equipped

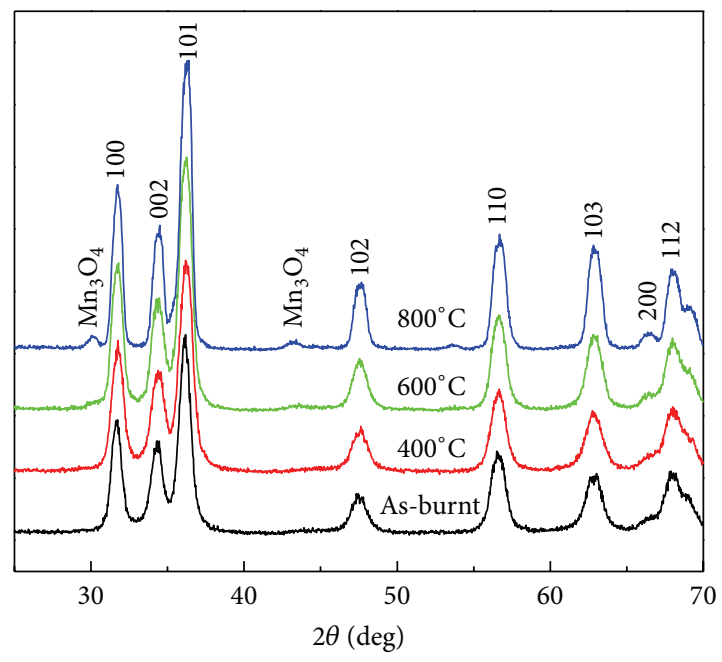

FIGURE 1: XRD spectra of as-burnt and annealed nanostructures of $\mathrm{Zn}_{0.95} \mathrm{Mn}_{0.05} \mathrm{O}$.

with energy dispersive X-ray (EDX) system for morphological and elemental compositional analysis, respectively. Temperature-dependent electrical resistivity was measured using the two-point probe setup. Magnetic properties of samples were obtained from a MPMS XL, Quantum Design, superconducting quantum interference device (SQUID).

\section{Results and Discussion}

3.1. X-Ray Diffraction (XRD) Studies. Figure 1 shows the XRD patterns of as-burnt and thermally annealed samples at 400, 600 , and $800^{\circ} \mathrm{C}$ for 8 hours in air. The XRD patterns reveal that $\mathrm{Mn}$ substitution does not disturb the hexagonal wurtzite type structure and space group of $\mathrm{P}_{3} \mathrm{mc}$ of the host $\mathrm{ZnO}$, similar to what has been realized in the previously reported works $[18,23]$. The peaks in the diffraction patterns turned out to be more intense and slightly shifted due to thermal annealing. The change in the lattice parameters occurs due to the crystalline refinement process during thermal treatment. However, some additional peak referring to the manganese oxide impurity phase $\left(\mathrm{Mn}_{3} \mathrm{O}_{4}\right)$ was detected in the sample annealed at $800^{\circ} \mathrm{C}$. No other impurity phase was observed in any other pattern. The calculated values of the lattice parameters $a$ and $c$ were observed to vary between 3.24893.2511 $\AA$ and 5.2005-5.2088 $\AA$, respectively.

Figure 2 demonstrates the variation of lattice parameters with variation of thermal annealing temperature. The average crystallite size was calculated by measuring the full width at half maximum (FWHM) of the most intense diffraction peak (101) in all the patterns using well-known Scherrer formula [7]:

$$
D_{h, k, l}=\frac{k \lambda}{\beta \cos \theta},
$$

where " $\theta$ " is Bragg's angle, " $\lambda$ " denotes the wavelength $(\lambda=$ $1.5405 \AA$ ) of $\mathrm{Cu} K_{\alpha}$ radiation, and " $D_{h, k, l}$ " depicts the average crystallite diameter and " $\beta$ " the full width at half maximum in 


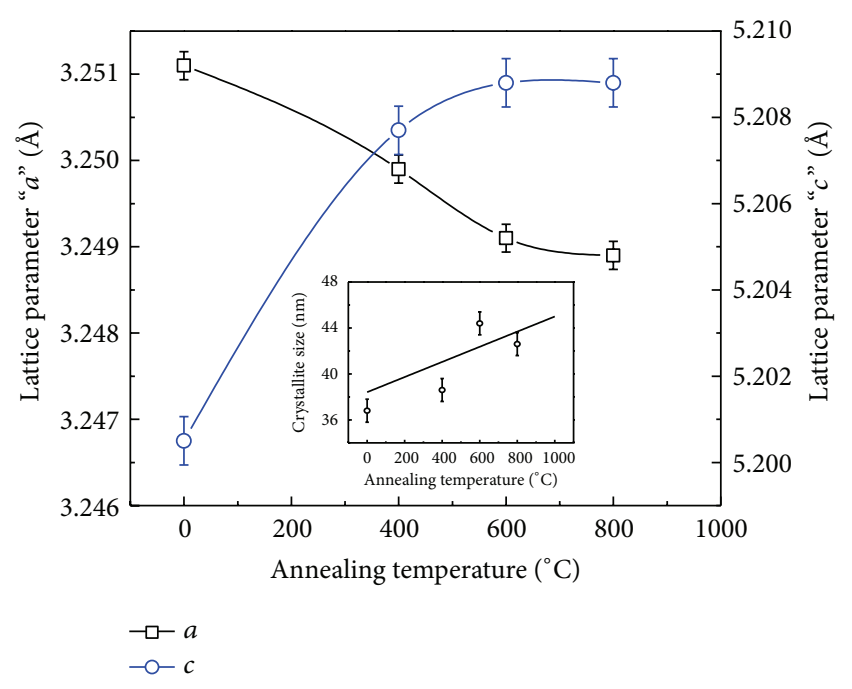

FIgURE 2: Variation in lattice parameters " $a$ " and " $c$ " and crystallite size (inset) with the increase in annealing treatment.

radians. The crystallite size of as-burnt sample was $36.77 \mathrm{~nm}$, which was found to vary as $44.40 \mathrm{~nm}$ with the rise in annealing temperature, as illustrated in Figure 2 (inset). The relative decrease in crystallite size at $800^{\circ} \mathrm{C}$ is attributed to the formation of the manganese oxide impurity phase.

3.2. FESEM and EDX Analysis. Figures 3(a)-3(c) show the FESEM micrograph of as synthesized and annealed samples of $\mathrm{Mn}$ doped $\mathrm{ZnO}$ nanostructures, respectively. As can be seen in Figure 3(a), the grain size of as synthesized sample is not uniform and turns into agglomerated clusters of highly refined nanometer sized grains when subjected to the thermal annealing treatment. A good proportion of the grains can be seen with small grain size under $100 \mathrm{~nm}$, whereas very large agglomerates having sizes of about $1-2 \mu \mathrm{m}$ are also present which can be collimated up to $50 \%$. The subsequent thermal annealing on as synthesized powder samples at 400 and $600^{\circ} \mathrm{C}$ has shown significantly modified morphology. The grains are showing well-defined shapes and edges. It can be inferred that the temperature of thermal treatment significantly affects the crystallinity and uniformity of the structure. The sample annealed at $600^{\circ} \mathrm{C}$ reveals uniform sized, highly ordered, and phase pure grain structure, which is consistent with the results obtained from XRD analysis. Figure 3(d) shows the EDX pattern revealing the wt\% of $\mathrm{Zn}, \mathrm{Mn}$, and $\mathrm{O}$ elements present in as-burnt $\mathrm{Zn}_{0.95} \mathrm{Mn}_{0.05} \mathrm{O}$ sample. The EDX analysis confirms the incorporation of manganese in the $\mathrm{ZnO}$ structure and wt $\%$ is very nearly equal to the nominal value of $\mathrm{Mn}$ in $\mathrm{ZnO}$. EDX quantitative data in Table 1 reveals that the $\mathrm{Zn}, \mathrm{Mn}$, and $\mathrm{O}$ contents are in quite close agreement with the stoichiometric compositions of the dissolved reactants. Traces of small wt $\%$ of $\mathrm{C}$ observed in the sample could be originating from the sample stub.

3.3. Electrical Resistivity Analysis. Volume resistance $\left(R_{v}\right)$ as a function of temperature of as-burnt and annealed samples
TABLE 1: EDX quantitative data of as-burnt $\mathrm{Zn}_{0.95} \mathrm{Mn}_{0.05} \mathrm{O}$.

\begin{tabular}{lcc}
\hline Element & Weight\% & Atomic\% \\
\hline $\mathrm{C}$ & 0.71 & 2.45 \\
$\mathrm{O}$ & 22.20 & 48.29 \\
$\mathrm{Mn}$ & 4.73 & 3.56 \\
$\mathrm{Zn}$ & 72.34 & 45.70 \\
Total & $\mathbf{1 0 0 . 0 0}$ & $\mathbf{1 0 0 . 0 0}$ \\
\hline
\end{tabular}

was evaluated and the corresponding DC electrical resistivity values were estimated using the relation $\rho_{v}=R_{v} A / L$ [24], where, $\rho_{v}, R_{v}, A$, and $L$ represent the volume resistivity, volume resistance, total area of the sample, and thickness of the pellet, respectively. The values of temperature-dependent DC electrical resistivity measured for all the samples are shown in Figure 4. The overall trend of resistivity decreased with rise of temperature depicting the semiconducting nature of compositions. The lower values of resistivity in annealed samples correspond to the crystalline refinement process associated with the removal of stresses and reduced effect of scattering at crystalline boundaries.

3.4. Magnetic Measurements. Temperature-dependent $M / H$ curves of the samples were obtained in the fixed field range of $10 \mathrm{kOe}$ as shown in Figure 5, indicating clear magnetization in as-burnt and annealed nanostructures. Ferromagnetic interactions in DMS materials can be explained by the RKKY theory [18, 25], according to which the magnetism arises as a result of the exchange interaction between local spinpolarized electrons (such as the electrons of $\mathrm{Mn}^{2+}$ ions) and conductive electrons $[18,26]$. Figure 5 (inset) shows the inverse susceptibility $\chi^{-1}$ plotted as a function of temperature. Extrapolation of the curves towards $x$-axis specifically at low temperature gives positive values of Weiss temperature revealing the ferromagnetic nature of material. Wang and coworkers [27] have attributed the origin of ferromagnetism in $\mathrm{Mn}$ doped $\mathrm{ZnO}$ DMS to the existence of secondary phases, like $\mathrm{Mn}_{3} \mathrm{O}_{4}$. However, the origin of room-temperature ferromagnetism in $\mathrm{ZnMnO}$ DMS is not completely clear yet. Recently, Yang and coworkers [28] confirmed that the origin of room-temperature magnetism could not derive from any impurity or secondary phase. In the meanwhile, decrease in magnetization in sample annealed at $800^{\circ} \mathrm{C}$ having clusters of impurity phases corroborates the absence of it having any role in the origination of magnetization. It appears that annealing temperature affects the structural arrangement, which in turn results in the improvement of the magnetic properties. The interactions between the $\mathrm{Mn}^{2+}$ atoms successfully substituted at the $\mathrm{Zn}^{2+}$ sites may contribute to the ferromagnetic behavior of $\mathrm{Mn}$ doped $\mathrm{ZnO}$.

It was observed from $M / H$ curves that ferromagnetic interactions appeared at room temperature and showed enhanced values as the temperature was decreased below room temperature. The maximum magnetization was observed at the lowest value oftemperature $(50 \mathrm{~K})$ which decreased with the rise in temperature. This is in accordance with the Weiss model of ferromagnetism according to which 


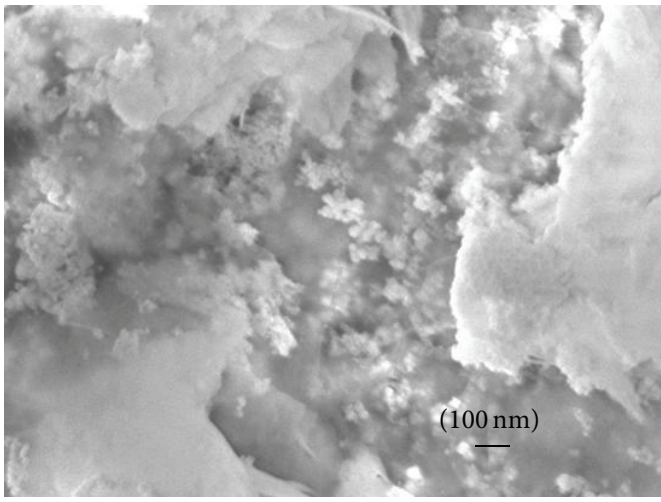

(a)

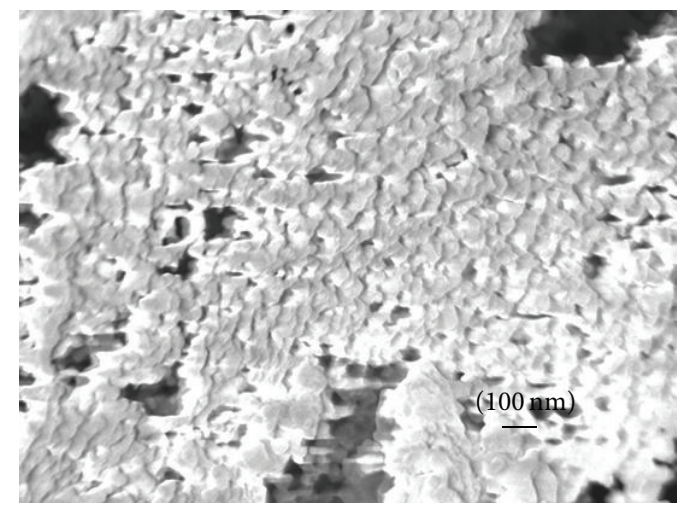

(c)

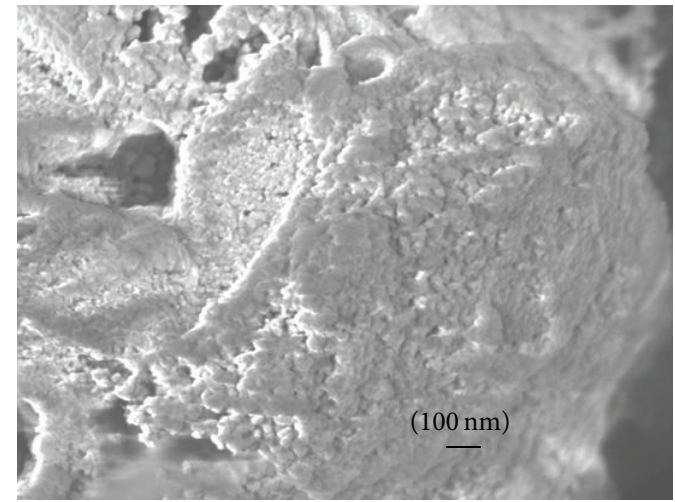

(b)

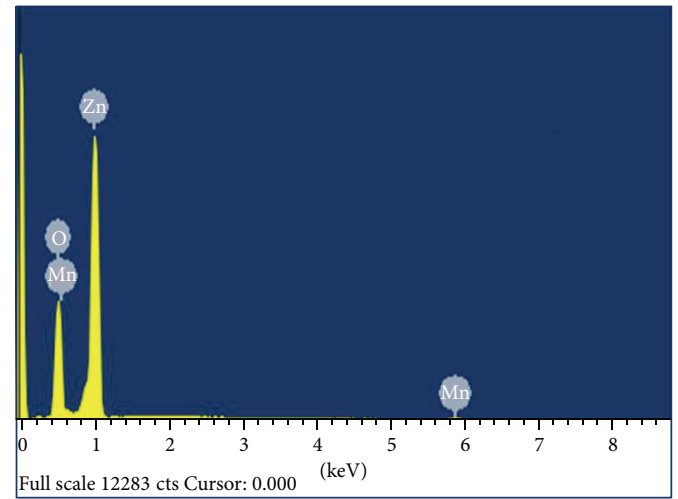

(d)

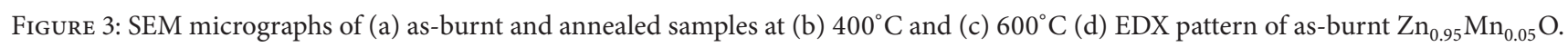

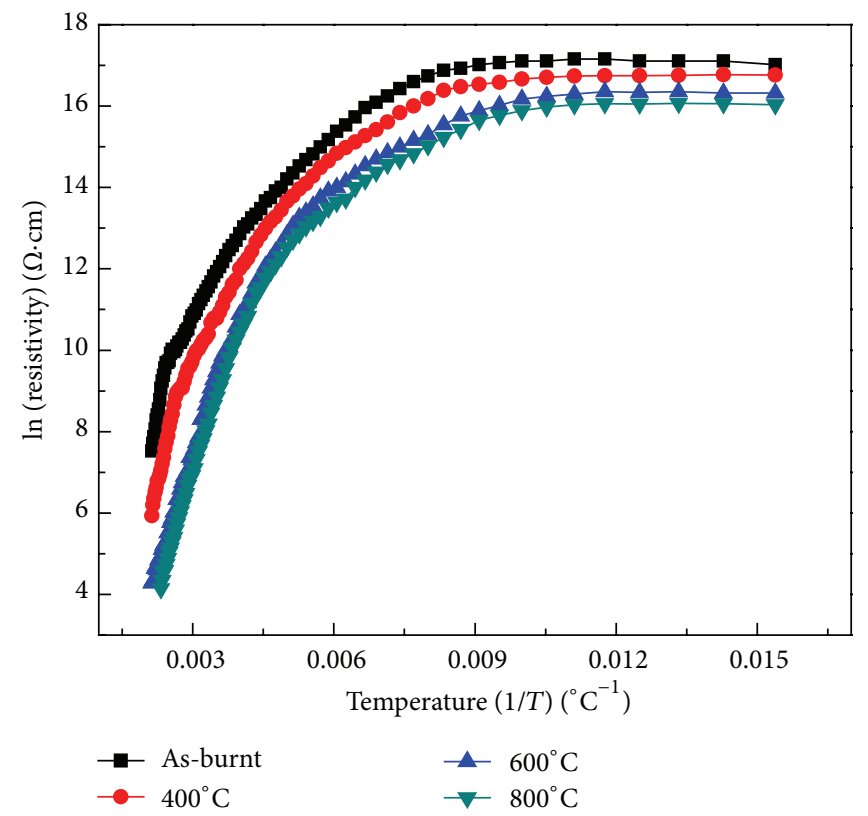

FIGURE 4: Temperature-dependent resistivity of as-burnt and annealed $\mathrm{Zn}_{0.95} \mathrm{Mn}_{0.05} \mathrm{O}$. 


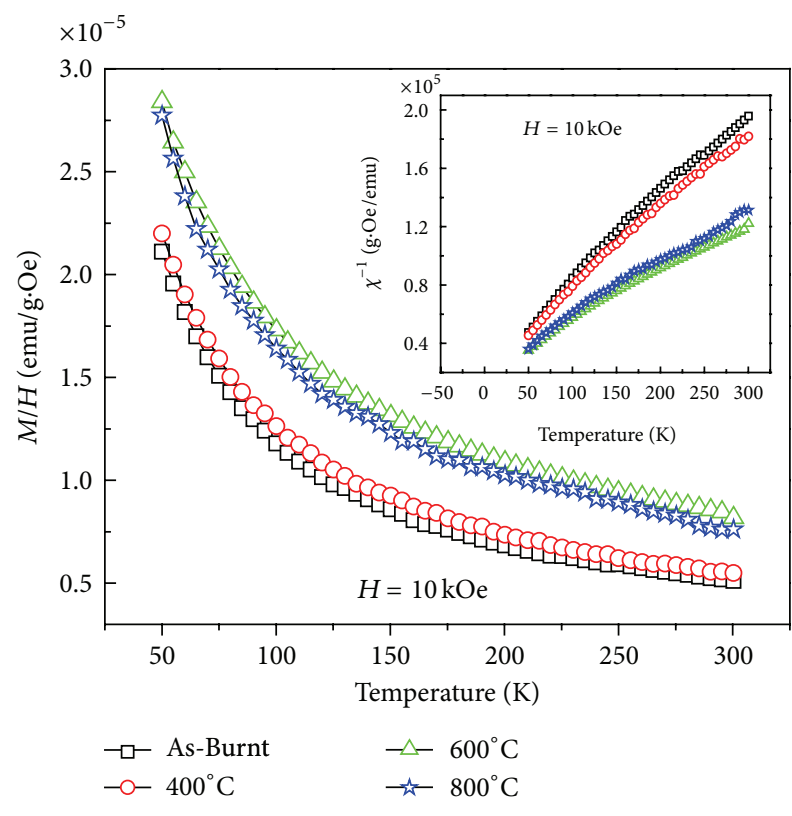

FIgURE 5: Temperature-dependent $M / H$ and (inset) inverse magnetic susceptibility $\left(\chi^{-1}\right)$ of as-burnt and annealed $\mathrm{Zn}_{0.95} \mathrm{Mn}_{0.05} \mathrm{O}$.

the thermal fluctuations begin to destroy the magnetization. The magnetization values of the samples were observed to increase for the samples annealed up to $600^{\circ} \mathrm{C}$. However, this value was decreased in the sample annealed at $800^{\circ} \mathrm{C}$. Once again, this is likely due to the presence of paramagnetic or diamagnetic islands of the impurity phases, corroborated by the structural analysis by XRD. Sharma and coworkers [18] reported that the lower values of saturation magnetization might be due to the smaller sizes of crystallites in the samples. As the value of crystallite size increased with the elevated thermal treatment up to $600^{\circ} \mathrm{C}$, the corresponding saturation magnetization also increased in concordance with reported results. Crystalline boundaries strongly influence the individual magnetic domain effect and hence are involved in possible interfacial scattering. Strong influence on the magnetization is attributed to low interfacial scattering due to increase in crystallite size with reduced crystalline boundaries.

\section{Conclusion}

Wurtzite type hexagonal structure was detected by XRD patterns in as-burnt and thermally annealed Mn doped $\mathrm{ZnO}$ nanostructures. The values of lattice parameters and crystallite size were changed with varying the annealing temperature due to improvement in morphology and $\mathrm{Mn}$ ion substitution at regular sites of $\mathrm{Zn}$. The electrical resistivity measurements confirmed the semiconducting nature of compositions. The magnetic measurements show the ferromagnetic interactions in as-burnt and thermally annealed samples. Thermal annealing affects the structural properties causing changes in saturation magnetization. The sample annealed at $800^{\circ} \mathrm{C}$ was found to have low magnetization due to the presence of paramagnetic or diamagnetic effects from manganese oxide impurity phase. Much improved results with respect to saturation magnetization were obtained in more structurally homogeneous composition. It was inferred from these investigations that structural morphology directly influenced the electrical and magnetic properties of DMS materials.

\section{Conflict of Interests}

The authors declare that there is no conflict of interests regarding the publication of this paper.

\section{Acknowledgments}

The authors would like to thank Higher Education Commission (HEC), Pakistan, for funding this research work under indigenous 5000 fellowships scheme. The authors extend their appreciation to the Deanship of Scientific Research at King Saud University for funding the work through the research group Project no. RGP-VPP-311.

\section{References}

[1] S. Qingbo, Z. Yuping, and J. Dongliang, "Doped ions $\left(\mathrm{Co}^{2+}\right.$, $\mathrm{Fe}^{3+}$ ) tuning morphologies and magnetic properties of indium oxide nanoparticles," Journal of Nanoparticle Research, vol. 14, article 655, 2012.

[2] J. Mera, C. Cordoba, C. Paucar, A. Gomez, D. Fuchs, and O. Moran, "On the structural analysis and magnetic behavior of heavily doped $\mathrm{Zn}_{1-x} \mathrm{Mn}_{x} \mathrm{O}$ nanopowders synthesized by wet chemistry method," Journal of Magnetism and Magnetic Materials, vol. 324, no. 10, pp. 1758-1763, 2012.

[3] J. Elanchezhiyan, K. P. Bhuvana, N. Gopalakrishnan, and T. Balasubramanian, "Investigation on $\mathrm{Mn}$ doped $\mathrm{ZnO}$ thin films grown by RF magnetron sputtering," Materials Letters, vol. 62, no. 19, pp. 3379-3381, 2008.

[4] L. B. Duan, W. G. Chu, J. Yu et al., "Structural and magnetic properties of $\mathrm{Zn}_{1-x} \mathrm{Co}_{x} \mathrm{O}(0<\mathbf{x} \leq 0.30)$ nanoparticles," Journal of Magnetism and Magnetic Materials, vol. 320, no. 8, pp. 15731581, 2008.

[5] A. S. Risbud, N. A. Spaldin, Z. Q. Chen, S. Stemmer, and R. Seshadri, "Magnetism in polycrystalline cobalt-substituted zinc oxide," Physical Review B, vol. 68, no. 20, Article ID 205202, 2003.

[6] V. K. Sharma and G. D. Varma, "Co nanoclusters as origin of ferromagnetism in sol-gel synthesized $\mathrm{Zn}_{1-x} \mathrm{Co}_{x} \mathrm{O}(\mathrm{x}=0.05$, 0.10 and 0.15) samples," Crystal Research and Technology, vol. 43, no. 10, pp. 1046-1051, 2008.

[7] G. Pei, F. Wu, C. Xia, J. Zhang, X. Li, and J. Xu, "Influences of $\mathrm{Al}$ doping concentration on structural, electrical and optical properties of $\mathrm{Zn}_{0.95} \mathrm{Ni}_{0.05} \mathrm{O}$ powders," Current Applied Physics, vol. 8, no. 1, pp. 18-23, 2008.

[8] C. Cheng, G. Xu, H. Zhang, Y. Luo, and Y. Li, "Solution synthesis, optical and magnetic properties of $\mathrm{Zn}_{1-x} \mathrm{Co}_{x} \mathrm{O}$ nanowires," Materials Letters, vol. 62, no. 21-22, pp. 3733-3736, 2008.

[9] S. W. Lim, M. C. Jeong, M. H. Ham, and J. M. Myoung, "Holemediated ferromagnetic properties in $\mathrm{Zn}_{1-x} \mathrm{Mn}_{x} \mathrm{O}$ thin films," Japanese Journal of Applied Physics, vol. 43, part 2, no. 2, article L280, 2004. 
[10] K. Ravichandran, K. Karthika, B. Sakthivel et al., "Tuning the combined magnetic and antibacterial properties of $\mathrm{ZnO}$ nanopowders through Mn doping for biomedical applications," Journal of Magnetism and Magnetic Materials, vol. 358-359, pp. 50-55, 2014.

[11] T. Dietl, H. Ohno, F. Matsukura, J. Cibert, and D. Ferrand, "Zener model description of ferromagnetism in zinc-blende magnetic semiconductors," Science, vol. 287, no. 5455, pp. 10191022, 2000.

[12] S. Chattopadhyay, S. K. Neogi, A. Sarkar et al., "Defects induced ferromagnetism in $\mathrm{Mn}$ doped $\mathrm{ZnO}$," Journal of Magnetism and Magnetic Materials, vol. 323, no. 3-4, pp. 363-368, 2011.

[13] K. Rode, A. Anane, R. Mattana, J.-P. Contour, O. Durand, and R. LeBourgeois, "Magnetic semiconductors based on cobalt substituted $\mathrm{ZnO}$," Journal of Applied Physics, vol. 93, no. 10, pp. 7676-7678, 2003.

[14] S. J. Han, T. H. Jang, Y. B. Kim, B. G. Park, J. H. Park, and Y. H. Jeong, "Magnetism in $\mathrm{Mn}$-doped $\mathrm{ZnO}$ bulk samples prepared by solid state reaction," Applied Physics Letters, vol. 83, no. 5, pp. 920-922, 2003.

[15] J. H. Li, D. Z. Shen, Y. J. Zhang et al., "Magnetism origin of Mndoped $\mathrm{ZnO}$ nanoclusters," Journal of Magnetism and Magnetic Materials, vol. 302, no. 1, pp. 118-121, 2006.

[16] P. Sharma, A. Gupta, K. V. Rao et al., "Ferromagnetism above room temperature in bulk and transparent thin films of Mndoped ZnO," Nature Materials, vol. 2, no. 10, pp. 673-677, 2003.

[17] W. Chen, L. F. Zhao, Y. Q. Wang et al., "Effects of temperature and atmosphere on the magnetism properties of Mn-doped ZnO," Applied Physics Letters, vol. 87, no. 4, Article ID 42507, 2005.

[18] V. K. Sharma, R. Xalxo, and G. D. Varma, "Structural and magnetic studies of Mn-doped ZnO," Crystal Research and Technology, vol. 42, no. 1, pp. 34-38, 2007.

[19] C. Sudakar, J. S. Thakur, G. Lawes, R. Nailk, and V. M. Naik, "Ferromagnetism induced by planar nanoscale $\mathrm{CuO}$ inclusions in Cu-doped $\mathrm{ZnO}$ thin films," Physical Review B, vol. 75, Article ID 054423, 2007.

[20] S. Zhou, K. Potzger, G. Zhang et al., "Crystalline Ni nanoparticles as the origin of ferromagnetism in $\mathrm{Ni}$ implanted $\mathrm{ZnO}$ crystals," Journal of Applied Physics, vol. 100, no. 11, Article ID 114304, 2006.

[21] C. Karunakaran, P. Vinayagamoorthy, and J. Jayabharathi, "Electrical, optical and photocatalytic properties of polyethylene glycol-assisted sol-gel synthesized $\mathrm{Mn}$-doped $\mathrm{TiO}_{2} / \mathrm{ZnO}$ core-shell nanoparticles," Superlattices and Microstructures, vol. 64, pp. 569-580, 2013.

[22] M. Vagadia, A. Ravalia, U. Khachar et al., "Size and grain morphology dependent magnetic behaviour of Co-doped $\mathrm{ZnO}$," Materials Research Bulletin, vol. 46, no. 11, pp. 1933-1937, 2011.

[23] S. Deka and P. A. Joy, "Synthesis and magnetic properties of Mn doped ZnO nanowires," Solid State Communications, vol. 142, no. 4, pp. 190-194, 2007.

[24] C. Kato, K. Kuroda, and K. Hasegawa, "Electrical conductivity of a montmorillonite-organic complex," Clay Minerals, vol. 14, p. 13, 1979.

[25] D. J. Priour Jr., E. H. Hwang, and S. das Sarma, "Disordered RKKY lattice mean field theory for ferromagnetism in diluted magnetic semiconductors," Physical Review Letters, vol. 92, no. 11, Article ID 117201, 2004.

[26] M. Saleem, S. A. Siddiqi, S. Atiq, M. S. Anwar, and S. Riaz, "Room temperature magnetic behavior of sol-gel synthesized
Mn doped ZnO,' Chinese Journal of Chemical Physics, vol. 23, no. 4, article 469, 2010.

[27] H. B. Wang, H. Wang, C. Zhang et al., "Effect of annealing on the magnetic properties of solution synthesized $\mathrm{Zn}_{1-x} \mathrm{Mn}_{x} \mathrm{O}$ nanorods," Materials Chemistry and Physics, vol. 113, no. 2-3, pp. 884-888, 2009.

[28] T. Yang, Y. Li, M. Y. Zhu et al., "Room-temperature ferromagnetic $\mathrm{Mn}$-doped $\mathrm{ZnO}$ nanocrystal synthesized by hydrothermal method under high magnetic field," Materials Science and Engineering B, vol. 170, no. 1-3, pp. 129-132, 2010. 

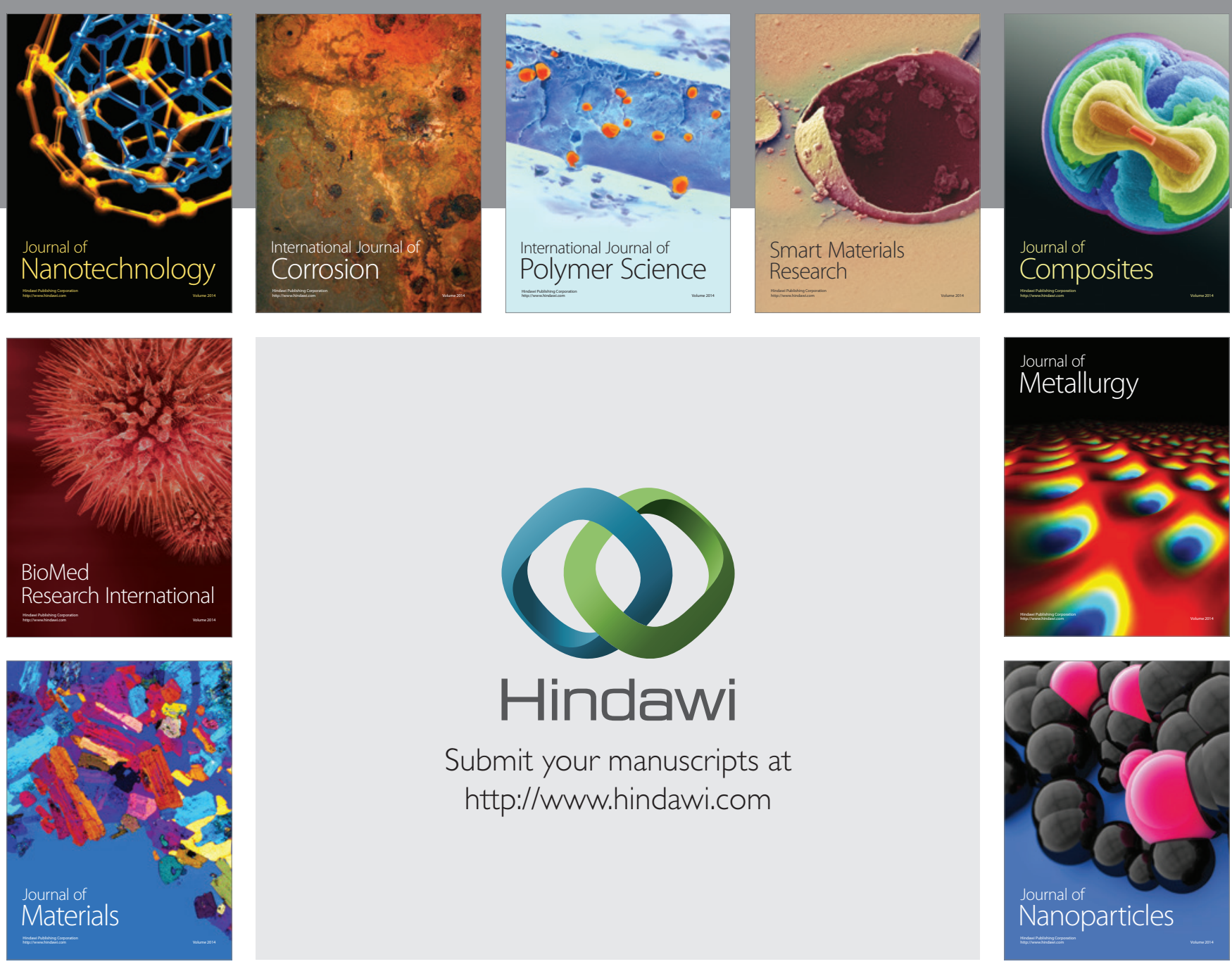

Submit your manuscripts at http://www.hindawi.com
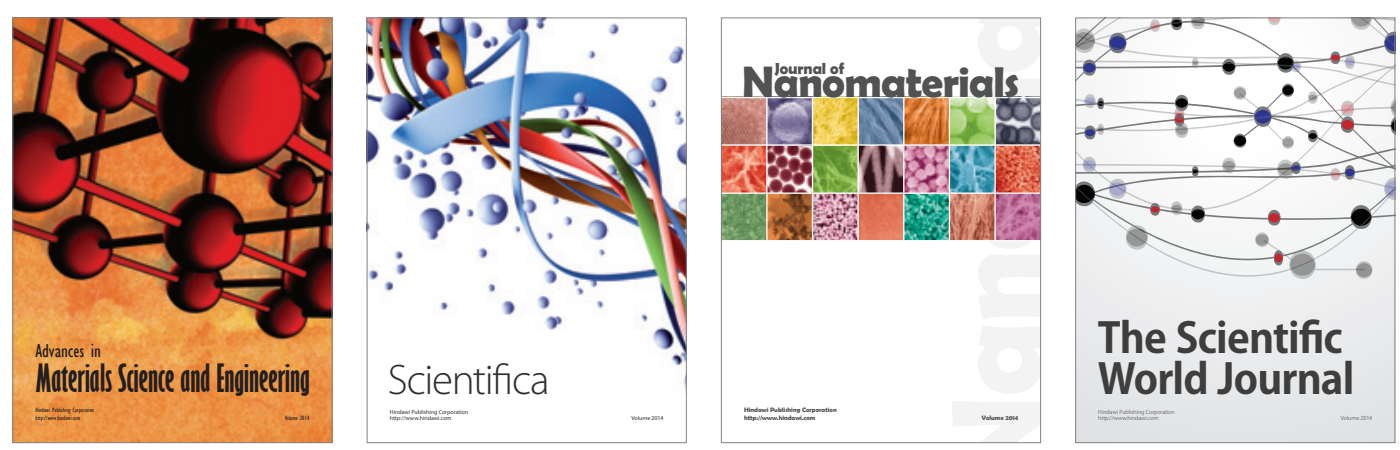

\section{The Scientific World Journal}
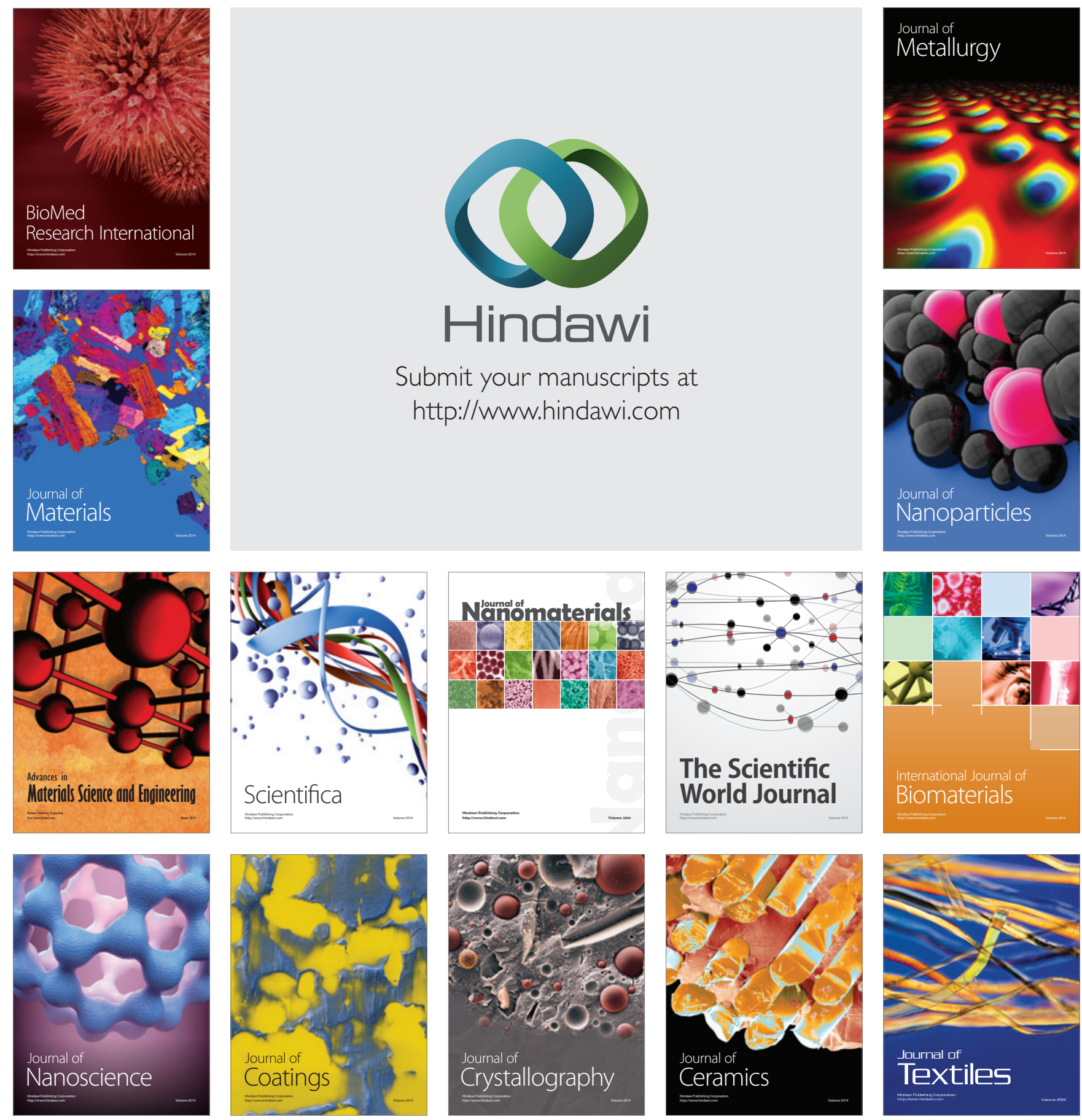